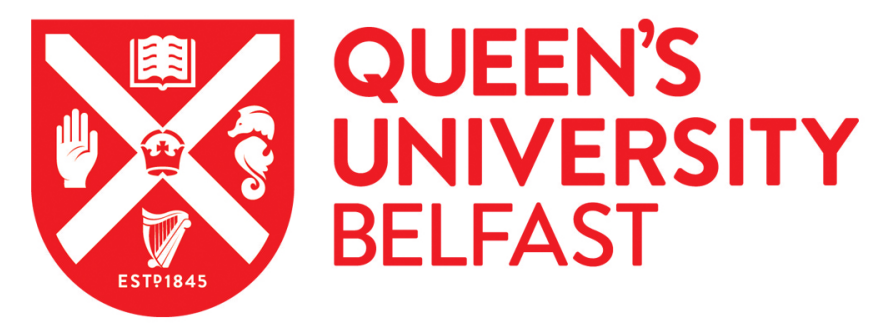

\title{
The Hubble Wide Field Camera 3 Test of Surfaces in the Outer Solar System: Spectral Variation on Kuiper Belt Objects
}

Fraser, W. C., Brown, M. E., \& Glass, F. (2015). The Hubble Wide Field Camera 3 Test of Surfaces in the Outer Solar System: Spectral Variation on Kuiper Belt Objects. The Astrophysical Journal, 804(1), [31]. https://doi.org/10.1088/0004-637X/804/1/31

\section{Published in:}

The Astrophysical Journal

Document Version:

Publisher's PDF, also known as Version of record

Queen's University Belfast - Research Portal:

Link to publication record in Queen's University Belfast Research Portal

Publisher rights

(c) 2015. The American Astronomical Society. All rights reserved.

This work is made available online in accordance with the publisher's policies.

\section{General rights}

Copyright for the publications made accessible via the Queen's University Belfast Research Portal is retained by the author(s) and / or other copyright owners and it is a condition of accessing these publications that users recognise and abide by the legal requirements associated with these rights.

Take down policy

The Research Portal is Queen's institutional repository that provides access to Queen's research output. Every effort has been made to ensure that content in the Research Portal does not infringe any person's rights, or applicable UK laws. If you discover content in the Research Portal that you believe breaches copyright or violates any law, please contact openaccess@qub.ac.uk. 


\title{
THE HUBBLE WIDE FIELD CAMERA 3 TEST OF SURFACES IN THE OUTER SOLAR SYSTEM: SPECTRAL VARIATION ON KUIPER BELT OBJECTS
}

\author{
Wesley C. Fraser ${ }^{1,2}$, Michael E. Brown ${ }^{2}$, and Florian Glass ${ }^{3,4}$ \\ ${ }^{1}$ Herzberg Institute of Astrophysics, 5071 West Saanich Road Victoria, BC V9E 2E7, Canada; wesley.fraser@nrc.ca \\ ${ }^{2}$ California Institute of Technology, 1200 East California Boulevard, Pasadena, CA 91101, USA \\ ${ }^{3}$ Observatoire de Genve, Universit de Genve, 51 chemin des Maillettes, CH-1290 Sauverny, Switzerland \\ ${ }^{4}$ Department of Physics and Astronomy, University of Victoria, Victoria, BC V8W 3P6, Canada \\ Received 2014 December 29; accepted 2015 February 23; published 2015 April 27
}

\begin{abstract}
Here, we present additional photometry of targets observed as part of the Hubble Wide Field Camera 3 (WFC3) Test of Surfaces in the Outer Solar System. Twelve targets were re-observed with the WFC3 in the optical and NIR wavebands designed to complement those used during the first visit. Additionally, all of the observations originally presented by Fraser and Brown were reanalyzed through the same updated photometry pipeline. A re-analysis of the optical and NIR color distribution reveals a bifurcated optical color distribution and only two identifiable spectral classes, each of which occupies a broad range of colors and has correlated optical and NIR colors, in agreement with our previous findings. We report the detection of significant spectral variations on five targets which cannot be attributed to photometry errors, cosmic rays, point-spread function or sensitivity variations, or other image artifacts capable of explaining the magnitude of the variation. The spectrally variable objects are found to have a broad range of dynamical classes and absolute magnitudes, exhibit a broad range of apparent magnitude variations, and are found in both compositional classes. The spectrally variable objects with sufficiently accurate colors for spectral classification maintain their membership, belonging to the same class at both epochs. 2005 TV189 exhibits a sufficiently broad difference in color at the two epochs that span the full range of colors of the neutral class. This strongly argues that the neutral class is one single class with a broad range of colors, rather than the combination of multiple overlapping classes.
\end{abstract}

Key words: Kuiper belt: general - planets and satellites: composition - techniques: photometric

\section{INTRODUCTION}

Small Kuiper Belt objects (KBOs) exhibit a broad range of colors, from completely neutral to some of the reddest objects in the Solar System (see, for example, Tegler et al. 2003). These objects fall into at least two distinct classes based on their surface colors (Tegler \& Romanishin 2003; Barucci et al. 2005; Fraser \& Brown 2012; Peixinho et al. 2012; Dalle Ore et al. 2013; Lacerda et al. 2014). These two classes are found throughout the Kuiper belt and centaur regions with no significant detected differences in the distribution of colors between different dynamical classes (Fraser \& Brown 2012; Peixinho et al. 2012; Dalle Ore et al. 2013). This suggests that the bimodal color distribution of KBOs and centaurs is not due to a current evolutionary process, but rather a primordial property of the entire population, and is likely a consequence of their compositions.

Two competing types of classification exist, based on the distribution and clumpiness of the observed colors. Fraser \& Brown (2012) find that the small KBOs bifurcate into only two classes, with each class occupying a continuum of optical and NIR colors. Barucci et al. (2005) and Dalle Ore et al. (2013) suggest an alternative, namely, that KBOs occupy a larger number of discrete classes with unique colors describing each class. Spectral variability holds the key to determining which of these classifications is correct. Consider an object that exhibits surface heterogeneity, and therefore spectral variability. The case in which an object belonging to a certain class exhibits a surface consistent with another class must be quite rare. Otherwise, that object will often be observed with intermediate colors between the two classes. If this were common to KBOs, then little clumping in the color distribution would be observed and the two separate classes would appear as one class of object in the global color distribution. Clearly, spectral variability has the potential to distinguish between the continuum classification and more discrete schemes.

Over the last decade, astronomers have reported various detections of spectrally variable KBOs. Two detections of surface heterogeneity from spectrophotometry are presented by Choi et al. (2003) and Lacerda et al. (2008). Lacerda et al. (2008) present the clear and confident identification of a red spot on the surface of KBO 136108, Haumea, which manifests itself as a variation in the observed optical and NIR colors correlated with Haumea's variable light curve. Choi et al. (2003) present VR photometry of object 1999 TD10. They find that TD10 exhibits wild optical color variations between one of the reddest objects in the known Kuiper Belt, and having colors significantly bluer than Solar. This detection seems questionable as such a large variation in color, if common to KBOs, should erase the bifurcated optical color distribution that has been so confidently detected. Luckily, 1999 TD10 belongs to the sample presented here.

Other reported heterogeneity comes from optical and NIR spectral observations of a few unique KBOs. Barucci et al. (2002) and later Merlin et al. (2005) and Licandro \& PinillaAlonso (2005) all reported variability in the water-ice absorption features exhibited by the Centaur 32532, Thereus. de Bergh et al. (2004) and Fornasier et al. (2004) reported variations in the optical spectral slopes of Plutinos 47932 and 38628-Huya. Fornasier et al. (2009) observed a lack of an absorption feature at $\sim 0.7 \mu \mathrm{m}$ in the optical spectrum of KBO 208996, 2003 AZ84, a feature detected in earlier spectra. Finally, Merlin et al. (2010) demonstrated that the optical and 
NIR photometry of Centaur Echeclus do not agree with its observed spectrum. Detections of surface heterogeneities from optical and NIR spectra are exceptionally challenging, often plagued by subtle calibration issues. These issues are notoriously difficult to diagnose, and can show themselves as "features" in the resultant spectra (see Bus et al. 2002 for a discussion of the difficulties in calibrating ground-based spectra). Unfortunately, to date, the detections of heterogeneity through spectra are all of a level similar to what may arise from calibration issues making their detection uncertain.

Here, we present optical and NIR spectro-photometry from the Wide Field Camera 3 (WFC3) on board the Hubble Space Telescope $(H S T)$ of $13 \mathrm{KBOs}$ along with past photometry presented by Fraser \& Brown (2012) taken as part of the Hubble/WFC3 Test of Surfaces in the Outer Solar System (H/ WTSOSS) and of target 2007 OR10 presented by Brown et al. (2011). Given the sensitivity stability of the HST (less than $1 \%$ variation in zero points for the cycles during which our observations were taken) and the avoidance of any atmospheric extinction calibration difficulties, the photometry we present here represent the best evidence to date of surface heterogeneity on a moderate sample of KBOs. In Section 2, we present our observations, photometry, and resultant coarse spectra. In Section 3, we present a discussion of the detection of light curve variability in eight of our targets, four of which also exhibit significant spectral variability, and conclude with a short discussion in Section 4.

\section{OBSERVATIONS AND REDUCTIONS}

Here, we present new observations of 13 KBOs taken with the WFC3 on board the HST during cycle 18 as part of H/ WTSOSS. The observations published in Fraser \& Brown (2012) were processed with version 2.7 of the CalWF3 processing pipeline. The pipeline has seen moderate improvement since, including slight changes to the photometric zero points and identification of additional bad pixels. A reanalysis of those data using pipeline version 3.1 was warranted. An improved photometry routine compared to that used in Fraser \& Brown (2012) was used on both the cycle 17 and cycle 18 data, and is described as follows.

\subsection{Cycle 18 Observations}

The photometry we present here were observed as part of cycle 18 under GO-Program 12234 as an extension of the H/ WTSOSS program (Fraser \& Brown 2012). Thirteen KBOs were targeted by this program. Targets were selected from the original program to create a sample spanning the full range of dynamical class, brightness, absolute magnitude, and color. The original purpose of this program was to gather spectral photometry in the wavelength regions between the filters observed in Fraser \& Brown (2012). F606w was repeated during cycle 18 to ensure that brightness normalization was possible between the separate cycle 17 and 18 visits. The original purpose of the program was to search for potential absorption features on faint KBOs. As will be demonstrated below, however, the main result of these observations is the clear detection of spectral variability on a number of our targets.

All targets were observed and reduced in an identical manner. Each target was observed during a single 44 minute observability window, during which the telescope was slewed at the apparent rate of motion of the target. During the window of observation, two images where acquired in each of the F606w (a), F775w (b), F098m (c), and F101w (d) filters, and four images were aquired in $\mathrm{F} 127 \mathrm{~m}(\mathrm{e})$. The images were taken in an ab-abcdee-cdee pattern with $2^{\prime \prime}$ dithers separating exposures, marked by the dashes. We utilized exposure times of $128 \mathrm{~s}, 114 \mathrm{~s}, 115 \mathrm{~s}, 207 \mathrm{~s}$, and $275 \mathrm{~s}$ in the F606w, F775w, F110w, F098m, and F127m filters, respectively. This observing pattern was chosen to afford some resilience to the effects of cosmic rays, and the possibility of detecting rotational variability during a sequence, all while ensuring that the data acquired could be fully downloaded during earth occultation.

We started with the non-multidrizzled CalWF3 v. 3.1 processed images. Full details of the pipeline are available in the CalWF3 manual, and include bias and dark subtraction, flat field corrections, pixel noise model generation, and conversion to flux units for the IR detector. An accurate centroid of the target $\mathrm{KBO}$ in each image was first determined. This was done by moving a 10 times oversampled theoretical TinyTim (Krist 1993) point-spread function (PSF) in a 0.01 pixel spacing grid in $\mathrm{x}$ and $\mathrm{y}$. The best-fit centroid was found as the coordinates which minimized the chi-square between the PSF and the observed KBO image. As the coordinates used by TinyTim do not match the photocenter of the theoretical PSF, the source photocenter was measured in a 4 pixel radius aperture about the PSF image at the best-fit coordinates. Finally, photometry was measured from the KBO using a circular aperture centered at the photocenter. This combination of steps was found to best minimize centroiding errors on faint sources. Aperture corrections were calculated from the theoretical PSF. Uncertainties were calculated by summing the pixels of the CalWF3 flux uncertainty extensions in each aperture using standard error propagation.

For all targets other than 1998 SM165, the photometry aperture used had a 4 pixel radius in all filters, corresponding to 0 0.16 and 0.52 in the UV and IR detectors, respectively. 1998 SM165 is a known binary that is partially resolved in the UV channel (F606w and F775w filters) and unresolved in the IR channel. A 9 pixel radius aperture was used to gather the flux from both sources. In the remaining filters, a 4 pixel radius aperture was used.

The magnitude in each filter was calculated as the mean of that filter's measurements. Visual inspection of the PSF radial profile after normalizing to the peak of each source was used to reject bad measurements; the profiles of each image pair were overlapped to allow easy identification of background sources and cosmic ray strikes which would affect the photometry. In addition, if the difference in magnitude between the two measurements of a pair was more than $3 \sigma$ discrepant, then the brighter of the two was rejected, as the most likely cause of dramatic apparent brightness variations is cosmic ray hits and background sources, both of which would be rejected by this procedure. An example of a rejected measurement can be seen in Figure 1. We report the cycle 18 photometry in the natural STMAG magnitude system in Table 1. As no phase curve information is available for our targets, we report absolute $\mathrm{H}_{606}$ magnitudes corrected for distance only.

Object 2001 QX322 was found to be offset from its predicted position at the time of observation, and as such fell on the edge of the optical images. As a result, no optical 


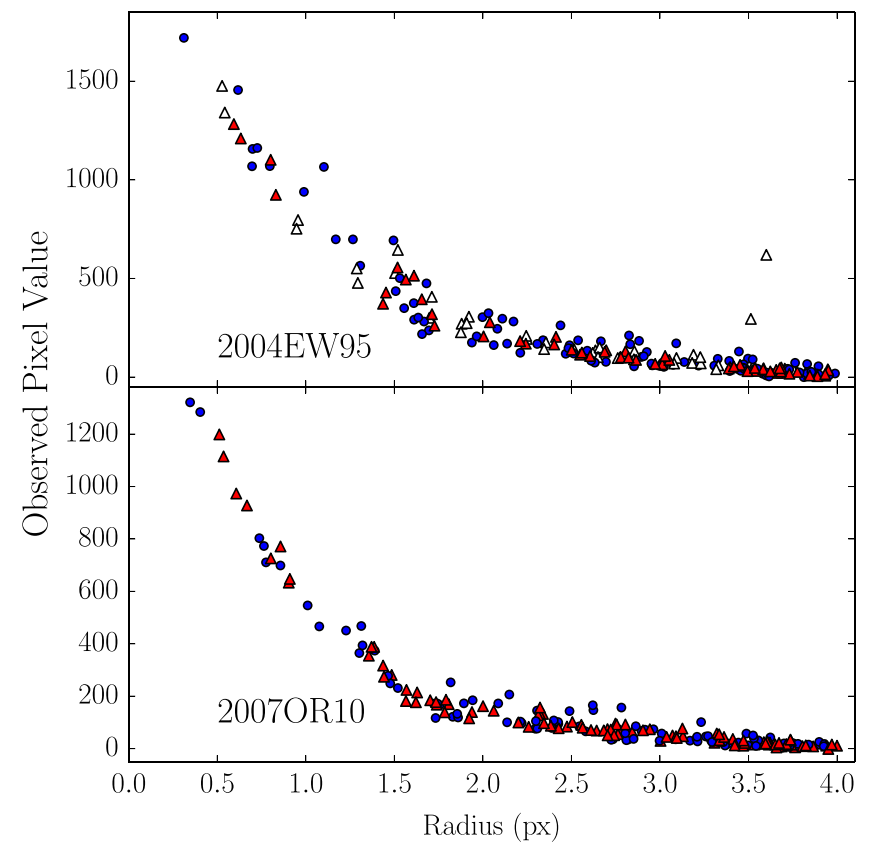

Figure 1. Measured radial PSFs of 2004 EW95 (top) and 2007 OR10 (bottom) in the F606w filter for observations taken in cycle 17 and 18, shown as blue circles and red triangles, respectively. One image of 2004 EW95 in cycle 18 was affected by a cosmic ray strike within the photometric aperture. The PSFs of that image are shown by the white points and the photometry from that image was rejected by our routine. The cosmic ray strike can be seen as higher than usual points in the white triangles at $\sim 3.5$ pixels radius.

photometry of 2001 QX322 are available from these observations.

\subsection{Cycle 17 Observations}

Using the techniques discussed above, we reanalyzed the cycle 17 photometry. For those sources with no obvious binary companions, 4 pixel radius apertures were used in the F606w and F814w filters, and 3.5 pixel radius apertures were used in the F139m and F153m filters.

For the known binaries 1998 SM165 and 2001 QC298, the secondaries were partially resolved in the optical images. For these objects, a single large aperture was used to gather the flux of both targets simultaneously. For the known binary 2003 HG57, a single aperture was used for both sources in the IR and two small apertures of 4 pixel radii were used in the optical, with appropriate aperture corrections applied. For binary 2001 QT297, the secondary was fully resolved in all images and small apertures were used accordingly. In all cases, we report the combined photometry of both sources. We will discuss resolved photometry in a separate manuscript.The re-analyzed photometry is presented in Table 2. Differences between the photometry presented in Fraser \& Brown (2012) and the reanalysis presented here are mainly due to better bad pixel identification and slight zero-point adjustments between versions 2.7 and 3.1 of the CalWF3 pipeline.

For 1998 SM165 we also consider the color (F606w$\mathrm{F} 814 \mathrm{w})=1.12 \pm 0.03$ in the Vega magnitude system reported by Benecchi et al. (2011) in our analysis of that target's spectral variability.

\subsection{Photometric Consistency}

The photometry we measure between the programs has proven to be consistent and repeatable for those objects which exhibit no significant light curves. This is demonstrated by objects 2007 OR10 and 2004 EW95, which have no more than 0.01 mag difference in F606w magnitude between separate visits of each object. This difference is consistent with the expected photometric precision, and is a result of WFC3's stable photometric sensitivity and our photometry routine.

The WFC3 photometric zero points have proven to be exceptionally stable over the time period in which our observations were taken. Kalirai et al. (2011) find less than $\sim 1$ variance of the zero points over $1.5 \mathrm{yr}$ of observations of four photometric standards.

Our procedure of first a visual rejection, then by magnitude difference proved excellent at rejecting those photometric measurements affected by cosmic rays or hot pixels. We demonstrate this with objects 2007 OR10 and 2004 EW95 in Figure 1.

For object 2007 OR10, background sources or cosmic ray strikes did not affect any of the four images taken in the F606w filter. It can be seen that the radial PSFs of all four images are very similar. All four measurements have a scatter about the mean of less than $0.01 \mathrm{mag}$, consistent with variation due to background noise, and were accepted by our photometry routine. This was not the case for 2004 EW95. Of the four images taken in the F606w filter, one was affected by either cosmic ray strikes or background sources within the photometric aperture. The radial profile allowed easy identification of

Table 1

Cycle 18 Spectral Photometry

\begin{tabular}{lccccrrr}
\hline \hline Designation $^{\mathrm{a}}$ & F606w & F775w & F110w & F098m & F127m & $H_{606}$ \\
\hline 26308 (1998 SM165) & $21.89 \pm 0.01$ & $22.04 \pm 0.02$ & $22.20 \pm 0.01$ & $22.02 \pm 0.01$ & $22.32 \pm 0.01$ & $6.13 \pm 0.01$ & $-0.11 \pm 0.02$ \\
137295 (1999 RB216) & $22.99 \pm 0.03$ & $23.16 \pm 0.05$ & $23.83 \pm 0.03$ & $23.48 \pm 0.03$ & $24.02 \pm 0.04$ & $7.70 \pm 0.03$ & $0.26 \pm 0.04$ \\
29981 (1999 TD10) & $22.56 \pm 0.01$ & $22.75 \pm 0.02$ & $23.41 \pm 0.02$ & $23.06 \pm 0.02$ & $23.52 \pm 0.02$ & $9.34 \pm 0.01$ & $0.57 \pm 0.02$ \\
2000 OH67 & $23.86 \pm 0.04$ & $24.05 \pm 0.06$ & $24.55 \pm 0.07$ & $24.25 \pm 0.10$ & $24.77 \pm 0.08$ & $7.49 \pm 0.04$ & $-0.29 \pm 0.06$ \\
2000 WT169 & $22.92 \pm 0.02$ & $22.91 \pm 0.04$ & $23.48 \pm 0.03$ & $23.23 \pm 0.03$ & $23.71 \pm 0.03$ & $6.41 \pm 0.02$ & $0.09 \pm 0.03$ \\
2001 PK47 & $23.49 \pm 0.04$ & $23.57 \pm 0.06$ & $24.10 \pm 0.03$ & $23.74 \pm 0.06$ & $24.20 \pm 0.05$ & $7.78 \pm 0.04$ & $0.00 \pm 0.05$ \\
2001 QX297 & $23.13 \pm 0.02$ & $23.27 \pm 0.04$ & $23.86 \pm 0.04$ & $23.54 \pm 0.05$ & $24.00 \pm 0.04$ & $6.81 \pm 0.02$ & $-0.28 \pm 0.03$ \\
2001 QX322 & $\ldots$ & $\ldots$ & $23.88 \pm 0.04$ & $23.53 \pm 0.03$ & $24.01 \pm 0.04$ & $\ldots$ & \\
2002 PV170 & $22.70 \pm 0.01$ & $22.76 \pm 0.02$ & $23.36 \pm 0.02$ & $23.00 \pm 0.03$ & $23.52 \pm 0.02$ & $6.49 \pm 0.01$ & $-0.11 \pm 0.02$ \\
120216 (2004 EW95) & $21.23 \pm 0.01$ & $21.57 \pm 0.01$ & $22.32 \pm 0.01$ & $21.97 \pm 0.01$ & $22.50 \pm 0.01$ & $6.80 \pm 0.01$ & $0.02 \pm 0.01$ \\
2004 QQ26 & $23.18 \pm 0.02$ & $23.38 \pm 0.04$ & $24.18 \pm 0.03$ & $23.90 \pm 0.06$ & $24.32 \pm 0.05$ & $10.1 \pm 0.02$ & $-0.06 \pm 0.03$ \\
2005 TV189 & $23.15 \pm 0.03$ & $23.40 \pm 0.04$ & $24.11 \pm 0.03$ & $23.85 \pm 0.04$ & $24.34 \pm 0.06$ & $8.12 \pm 0.03$ & $0.17 \pm 0.03$ \\
225088 (2007 OR10) & $21.66 \pm 0.01$ & $21.36 \pm 0.01$ & $21.699 \pm 0.007$ & $21.45 \pm 0.01$ & $21.826 \pm 0.008$ & $2.32 \pm 0.01$ & $-0.00 \pm 0.01$ \\
\hline
\end{tabular}


Table 2

Cycle 17 Spectral Photometry

\begin{tabular}{|c|c|c|c|c|c|c|c|c|}
\hline Designation $^{\mathrm{a}}$ & $a(\mathrm{AU})$ & $i\left(\left(^{\mathrm{o}}\right)\right.$ & $e$ & F606w & F814w & F139m & F153m & $\mathrm{H}_{606}$ \\
\hline Nessus (1993 HA2) & 24.47 & 15.65 & 0.51 & $23.32 \pm 0.02$ & $23.18 \pm 0.02$ & $24.04 \pm 0.04$ & $24.39 \pm 0.04$ & $9.82 \pm 0.02$ \\
\hline Hylonome (1995 DW2) & 24.99 & 4.14 & 0.24 & $23.41 \pm 0.03$ & $23.77 \pm 0.04$ & $24.60 \pm 0.07$ & $24.92 \pm 0.08$ & $10.12 \pm 0.04$ \\
\hline 26308 (1998 SM165) & 47.68 & 13.50 & 0.36 & $22.00 \pm 0.02$ & $22.06 \pm 0.02$ & $22.80 \pm 0.02$ & $22.99 \pm 0.02$ & $6.18 \pm 0.02$ \\
\hline Cyllarus (1998 TF35) & 26.27 & 12.63 & 0.37 & $22.48 \pm 0.01$ & $22.42 \pm 0.01$ & $23.26 \pm 0.03$ & $23.56 \pm 0.03$ & $8.82 \pm 0.01$ \\
\hline 69986 (1998 WW24) & 39.63 & 13.92 & 0.22 & $23.86 \pm 0.05$ & $24.01 \pm 0.04$ & $24.88 \pm 0.13$ & $25.13 \pm 0.10$ & $8.68 \pm 0.04$ \\
\hline 1998 WY24 & 43.45 & 1.90 & 0.04 & $23.57 \pm 0.03$ & $23.70 \pm 0.04$ & $24.59 \pm 0.07$ & $24.86 \pm 0.08$ & $7.39 \pm 0.04$ \\
\hline 1999 CL119 & 47.15 & 23.24 & 0.01 & $23.06 \pm 0.02$ & $23.23 \pm 0.02$ & $24.39 \pm 0.06$ & $24.67 \pm 0.06$ & $6.40 \pm 0.02$ \\
\hline 40314 (1999 KR16) & 48.73 & 24.81 & 0.30 & $21.61 \pm 0.01$ & $21.52 \pm 0.01$ & $22.35 \pm 0.01$ & $22.69 \pm 0.01$ & $6.11 \pm 0.01$ \\
\hline 137295 (1999 RB216) & 47.58 & 12.69 & 0.29 & $22.72 \pm 0.01$ & $22.88 \pm 0.02$ & $23.92 \pm 0.03$ & $24.20 \pm 0.04$ & $7.49 \pm 0.02$ \\
\hline 1999 RJ215 & 59.23 & 19.73 & 0.41 & $23.06 \pm 0.02$ & $23.26 \pm 0.02$ & $24.14 \pm 0.06$ & $24.42 \pm 0.05$ & $7.71 \pm 0.02$ \\
\hline 86177 (1999 RY215) & 45.18 & 22.21 & 0.23 & $22.82 \pm 0.02$ & $23.24 \pm 0.02$ & $24.23 \pm 0.05$ & $24.41 \pm 0.06$ & $7.31 \pm 0.02$ \\
\hline 91554 (1999 RZ215) & 101.0 & 25.54 & 0.69 & $23.14 \pm 0.02$ & $23.38 \pm 0.03$ & $24.24 \pm 0.06$ & $24.54 \pm 0.08$ & $7.98 \pm 0.03$ \\
\hline 29981 (1999 TD10) & 97.79 & 5.95 & 0.87 & $21.99 \pm 0.01$ & $22.27 \pm 0.01$ & $23.20 \pm 0.03$ & $23.50 \pm 0.03$ & $9.04 \pm 0.01$ \\
\hline Elatus (1999 UG5) & 11.81 & 5.24 & 0.38 & $22.45 \pm 0.01$ & $22.51 \pm 0.01$ & $23.68 \pm 0.04$ & $23.93 \pm 0.05$ & $10.93 \pm 0.01$ \\
\hline $121725(1999$ XX143) & 17.98 & 6.76 & 0.45 & $23.25 \pm 0.02$ & $23.39 \pm 0.03$ & $24.50 \pm 0.06$ & $24.79 \pm 0.06$ & $9.11 \pm 0.03$ \\
\hline 2000 AF255 & 48.89 & 30.87 & 0.24 & $23.37 \pm 0.02$ & $23.23 \pm 0.02$ & $23.96 \pm 0.04$ & $24.43 \pm 0.04$ & $6.30 \pm 0.02$ \\
\hline 2000 CE105 & 44.15 & 0.55 & 0.06 & $23.91 \pm 0.03$ & $23.92 \pm 0.04$ & $24.94 \pm 0.09$ & $25.06 \pm 0.13$ & $7.77 \pm 0.04$ \\
\hline $2000 \mathrm{CQ} 105$ & 57.36 & 19.64 & 0.39 & $23.28 \pm 0.02$ & $23.68 \pm 0.03$ & $24.62 \pm 0.07$ & $24.97 \pm 0.10$ & $6.56 \pm 0.03$ \\
\hline $60608(2000 \mathrm{EE} 173)^{\mathrm{b}}$ & 49.61 & 5.94 & 0.54 & $21.83 \pm 0.1$ & $22.09 \pm 0.1$ & $23.32 \pm 0.02$ & $23.61 \pm 0.02$ & $8.2 \pm 0.1$ \\
\hline 60620 (2000 FD8) & 43.80 & 19.49 & 0.21 & $22.90 \pm 0.02$ & $22.91 \pm 0.02$ & $23.54 \pm 0.04$ & $23.94 \pm 0.03$ & $7.08 \pm 0.02$ \\
\hline 2000 FV53 & 39.18 & 17.33 & 0.16 & $23.30 \pm 0.02$ & $23.74 \pm 0.04$ & $24.64 \pm 0.07$ & $24.81 \pm 0.07$ & $8.06 \pm 0.04$ \\
\hline 130391 (2000 JG81) & 47.37 & 23.46 & 0.27 & $23.75 \pm 0.04$ & $24.00 \pm 0.04$ & $24.72 \pm 0.08$ & $25.02 \pm 0.09$ & $8.33 \pm 0.04$ \\
\hline 2000 OH67 & 44.02 & 5.62 & 0.01 & $24.15 \pm 0.04$ & $24.21 \pm 0.05$ & $25.05 \pm 0.15$ & $25.42 \pm 0.17$ & $7.79 \pm 0.05$ \\
\hline 87555 (2000 QB243) & 34.78 & 6.79 & 0.56 & $23.08 \pm 0.03$ & $23.30 \pm 0.03$ & $24.22 \pm 0.05$ & $24.44 \pm 0.06$ & $9.14 \pm 0.03$ \\
\hline 123509 (2000 WK183) & 44.61 & 1.96 & 0.04 & $23.13 \pm 0.03$ & $23.26 \pm 0.03$ & $24.25 \pm 0.04$ & $24.49 \pm 0.05$ & $6.84 \pm 0.03$ \\
\hline 2000 WT169 & 45.11 & 1.74 & 0.00 & $22.82 \pm 0.02$ & $22.94 \pm 0.02$ & $23.83 \pm 0.06$ & $24.14 \pm 0.06$ & $6.32 \pm 0.02$ \\
\hline 2000 YB2 & 38.90 & 3.81 & 0.02 & $23.06 \pm 0.02$ & $23.28 \pm 0.04$ & $24.07 \pm 0.06$ & $24.45 \pm 0.05$ & $7.21 \pm 0.04$ \\
\hline $2000 \mathrm{YH} 2$ & 39.54 & 12.90 & 0.30 & $22.66 \pm 0.02$ & $23.01 \pm 0.02$ & $24.28 \pm 0.10$ & $24.40 \pm 0.05$ & $8.30 \pm 0.02$ \\
\hline 63252 (2001 BL41) & 9.725 & 12.49 & 0.29 & $22.99 \pm 0.03$ & $23.35 \pm 0.04$ & $24.05 \pm 0.04$ & $24.32 \pm 0.04$ & $12.06 \pm 0.04$ \\
\hline $150642(2001 \mathrm{CZ} 31)$ & 45.48 & 10.19 & 0.12 & $22.21 \pm 0.01$ & $22.60 \pm 0.01$ & $23.60 \pm 0.03$ & $23.86 \pm 0.03$ & $6.13 \pm 0.01$ \\
\hline 131318 (2001 FL194) & 39.41 & 13.68 & 0.17 & $23.30 \pm 0.02$ & $23.65 \pm 0.03$ & $24.71 \pm 0.09$ & $24.81 \pm 0.10$ & $8.13 \pm 0.03$ \\
\hline 2001 FO185 & 46.53 & 10.63 & 0.12 & $\ldots$ & $\ldots$ & $23.99 \pm 0.06$ & $25.05 \pm 0.16$ & $\ldots$ \\
\hline 82158 (2001 FP185) & 220.6 & 30.77 & 0.84 & $\ldots$ & $\ldots$ & $23.03 \pm 0.01$ & $23.33 \pm 0.02$ & $\ldots$ \\
\hline 2001 FQ185 & 47.78 & 3.23 & 0.23 & $23.25 \pm 0.02$ & $23.16 \pm 0.02$ & $24.11 \pm 0.04$ & $24.34 \pm 0.04$ & $7.61 \pm 0.02$ \\
\hline 82155 (2001 FZ173) & 85.79 & 12.70 & 0.62 & $\ldots$ & $\ldots$ & $22.87 \pm 0.01$ & $23.17 \pm 0.01$ & $\ldots$ \\
\hline 2001 HY 65 & 43.28 & 17.12 & 0.12 & $22.64 \pm 0.01$ & $22.76 \pm 0.02$ & $23.76 \pm 0.04$ & $24.05 \pm 0.05$ & $6.69 \pm 0.02$ \\
\hline $2001 \mathrm{KA} 77$ & 47.28 & 11.93 & 0.09 & $22.61 \pm 0.01$ & $22.55 \pm 0.01$ & $23.39 \pm 0.03$ & $23.67 \pm 0.03$ & $5.78 \pm 0.01$ \\
\hline 88269 (2001 KF77) & 25.91 & 4.35 & 0.23 & $23.84 \pm 0.04$ & $23.77 \pm 0.04$ & $24.51 \pm 0.12$ & $24.99 \pm 0.12$ & $10.71 \pm 0.04$ \\
\hline $88268(2001$ KK76) & 42.37 & 1.88 & 0.01 & $23.33 \pm 0.02$ & $23.32 \pm 0.03$ & $24.14 \pm 0.05$ & $24.38 \pm 0.06$ & $7.09 \pm 0.03$ \\
\hline 160147 (2001 KN76) & 43.68 & 2.64 & 0.08 & $23.33 \pm 0.02$ & $23.50 \pm 0.03$ & $24.56 \pm 0.10$ & $24.73 \pm 0.06$ & $7.33 \pm 0.03$ \\
\hline 2001 OQ108 & 45.52 & 2.32 & 0.01 & $23.82 \pm 0.03$ & $23.81 \pm 0.04$ & $24.67 \pm 0.10$ & $24.97 \pm 0.08$ & $7.26 \pm 0.04$ \\
\hline 2001 PK47 & 39.80 & 8.72 & 0.06 & $23.48 \pm 0.02$ & $23.78 \pm 0.05$ & $24.64 \pm 0.06$ & $24.76 \pm 0.06$ & $7.75 \pm 0.05$ \\
\hline 2001 QC298 & 46.19 & 30.62 & 0.12 & $22.63 \pm 0.02$ & $23.15 \pm 0.03$ & $24.15 \pm 0.05$ & $24.43 \pm 0.06$ & $6.59 \pm 0.03$ \\
\hline 139775 (2001 QG298) & 39.36 & 6.49 & 0.19 & $22.56 \pm 0.01$ & $22.62 \pm 0.01$ & $23.57 \pm 0.03$ & $23.85 \pm 0.04$ & $7.61 \pm 0.01$ \\
\hline 2001 QR297 & 44.25 & 5.15 & 0.02 & $23.47 \pm 0.03$ & $23.53 \pm 0.04$ & $24.39 \pm 0.07$ & $24.61 \pm 0.10$ & $7.07 \pm 0.04$ \\
\hline 2001 QR322 & 30.24 & 1.32 & 0.02 & $23.23 \pm 0.02$ & $23.56 \pm 0.03$ & $24.47 \pm 0.06$ & $24.76 \pm 0.06$ & $8.52 \pm 0.03$ \\
\hline 2001 QS322 & 43.96 & 0.24 & 0.03 & $23.64 \pm 0.03$ & $23.72 \pm 0.03$ & $24.72 \pm 0.10$ & $24.87 \pm 0.13$ & $7.40 \pm 0.03$ \\
\hline $\begin{array}{l}\text { Teharonhiawako } \\
\text { (2001 QT297) }\end{array}$ & 43.89 & 2.58 & 0.02 & $22.856 \pm 0.02$ & $22.904 \pm 0.02$ & $24.020 \pm 0.07$ & $24.308 \pm 0.05$ & $6.47 \pm 0.02$ \\
\hline 2001 QX297 & 44.01 & 0.91 & 0.02 & $23.42 \pm 0.02$ & $23.48 \pm 0.04$ & $24.39 \pm 0.08$ & $24.56 \pm 0.09$ & $7.06 \pm 0.04$ \\
\hline 2001 QX322 & 58.01 & 28.50 & 0.38 & $22.97 \pm 0.02$ & $23.17 \pm 0.02$ & $24.13 \pm 0.04$ & $24.46 \pm 0.05$ & $6.85 \pm 0.02$ \\
\hline 2001 RW143 & 43.06 & 2.96 & 0.03 & $23.91 \pm 0.04$ & $24.05 \pm 0.05$ & $\ldots$ & $\ldots$ & $7.73 \pm 0.05$ \\
\hline 2001 RZ143 & 44.12 & 2.12 & 0.06 & $23.03 \pm 0.02$ & $23.14 \pm 0.02$ & $24.07 \pm 0.06$ & $24.29 \pm 0.06$ & $6.93 \pm 0.02$ \\
\hline 119315 (2001 SQ73) & 17.50 & 17.43 & 0.17 & $22.04 \pm 0.01$ & $22.34 \pm 0.01$ & $23.31 \pm 0.03$ & $23.54 \pm 0.03$ & $9.65 \pm 0.01$ \\
\hline 2001 UP18 & 47.79 & 1.17 & 0.07 & $\ldots$ & $\ldots$ & $24.53 \pm 0.06$ & $24.82 \pm 0.07$ & $\ldots$ \\
\hline 148975 (2001 XA255) & 28.92 & 12.62 & 0.67 & $21.48 \pm 0.01$ & $21.78 \pm 0.01$ & $22.77 \pm 0.02$ & $22.97 \pm 0.02$ & $11.72 \pm 0.01$ \\
\hline 126155 (2001 YJ140) & 39.74 & 5.96 & 0.29 & $22.22 \pm 0.01$ & $22.49 \pm 0.01$ & $23.35 \pm 0.02$ & $23.57 \pm 0.02$ & $7.78 \pm 0.01$ \\
\hline 2002 CU154 & 44.02 & 3.34 & 0.06 & $23.66 \pm 0.03$ & $23.72 \pm 0.04$ & $24.67 \pm 0.06$ & $25.07 \pm 0.08$ & $7.56 \pm 0.04$ \\
\hline 2002 FW36 & 43.09 & 2.35 & 0.02 & $\ldots$ & $\ldots$ & $24.90 \pm 0.11$ & $25.11 \pm 0.13$ & $\ldots$ \\
\hline Crantor (2002 GO9) & 19.35 & 12.79 & 0.27 & $21.09 \pm 0.01$ & $21.16 \pm 0.01$ & $21.83 \pm 0.01$ & $22.13 \pm 0.01$ & $9.18 \pm 0.01$ \\
\hline 2002 GY32 & 39.44 & 1.80 & 0.09 & $23.32 \pm 0.02$ & $23.40 \pm 0.03$ & $24.36 \pm 0.05$ & $24.60 \pm 0.06$ & $7.78 \pm 0.03$ \\
\hline 2002 PD155 & 43.14 & 5.78 & 0.00 & $23.99 \pm 0.04$ & $24.14 \pm 0.07$ & $24.80 \pm 0.14$ & $25.42 \pm 0.16$ & $7.70 \pm 0.07$ \\
\hline 2002 PV170 & 42.55 & 1.27 & 0.01 & $22.81 \pm 0.02$ & $22.88 \pm 0.02$ & $23.85 \pm 0.04$ & $24.09 \pm 0.04$ & $6.54 \pm 0.02$ \\
\hline
\end{tabular}


Table 2

(Continued)

\begin{tabular}{|c|c|c|c|c|c|c|c|c|}
\hline Designation $^{\mathrm{a}}$ & $a(\mathrm{AU})$ & $i\left(^{\mathrm{o}}\right)$ & $e$ & F606w & F814w & $\mathrm{F} 139 \mathrm{~m}$ & F153m & $\mathrm{H}_{606}$ \\
\hline 2002 QX47 & 25.38 & 7.26 & 0.37 & & & $23.42 \pm 0.02$ & $23.67 \pm 0.02$ & \\
\hline 119976 (2002 VR130) & 24.07 & 3.52 & 0.38 & $23.47 \pm 0.03$ & $23.88 \pm 0.04$ & $24.65 \pm 0.07$ & $25.04 \pm 0.08$ & $11.72 \pm 0.04$ \\
\hline 119979 (2002 WC19) & 48.19 & 9.16 & 0.26 & $21.151 \pm 0.009$ & $21.24 \pm 0.01$ & $22.10 \pm 0.01$ & $22.38 \pm 0.01$ & $4.88 \pm 0.009$ \\
\hline 127546 (2002 XU93) & 67.50 & 77.89 & 0.68 & $21.71 \pm 0.01$ & $22.08 \pm 0.01$ & $23.07 \pm 0.01$ & $23.29 \pm 0.01$ & $8.49 \pm 0.01$ \\
\hline 2003 FE128 & 47.95 & 3.38 & 0.25 & $22.31 \pm 0.01$ & $22.39 \pm 0.02$ & $23.38 \pm 0.02$ & $23.70 \pm 0.02$ & $6.81 \pm 0.02$ \\
\hline 2003 FF128 & 39.47 & 1.91 & 0.21 & $22.34 \pm 0.01$ & $22.38 \pm 0.01$ & $23.38 \pm 0.03$ & $23.63 \pm 0.02$ & $7.31 \pm 0.01$ \\
\hline Ceto (2003 FX128) & 101.9 & 22.27 & 0.82 & $21.85 \pm 0.01$ & $22.07 \pm 0.01$ & $22.95 \pm 0.01$ & $23.25 \pm 0.01$ & $6.89 \pm 0.01$ \\
\hline 385437 (2003 GH55) & 44.16 & 1.10 & 0.08 & $22.63 \pm 0.01$ & $22.69 \pm 0.02$ & $23.49 \pm 0.03$ & $23.80 \pm 0.04$ & $6.53 \pm 0.02$ \\
\hline 2003 HG57 & 43.67 & 2.09 & 0.03 & $23.04 \pm 0.03$ & $23.31 \pm 0.05$ & $23.84 \pm 0.11$ & $24.04 \pm 0.12$ & $5.79 \pm 0.05$ \\
\hline 2003 LD9 & 47.12 & 6.98 & 0.17 & $23.38 \pm 0.02$ & $23.36 \pm 0.03$ & $24.41 \pm 0.05$ & $24.61 \pm 0.06$ & $7.23 \pm 0.03$ \\
\hline 2003 QA92 & 38.12 & 3.43 & 0.05 & $22.60 \pm 0.01$ & $22.69 \pm 0.02$ & $23.56 \pm 0.02$ & $23.83 \pm 0.03$ & $6.90 \pm 0.02$ \\
\hline 385447 (2003 QF113) & 43.67 & 4.46 & 0.02 & $23.60 \pm 0.03$ & $23.65 \pm 0.03$ & $24.66 \pm 0.10$ & $24.88 \pm 0.08$ & $7.34 \pm 0.03$ \\
\hline 2003 QX91 & 43.60 & 27.71 & 0.24 & $24.31 \pm 0.05$ & $24.56 \pm 0.10$ & $25.17 \pm 0.19$ & $25.51 \pm 0.20$ & $9.1 \pm 0.10$ \\
\hline 149560 (2003 QZ91) & 41.37 & 34.86 & 0.47 & $23.20 \pm 0.03$ & $23.43 \pm 0.03$ & $24.73 \pm 0.07$ & $24.90 \pm 0.08$ & $8.87 \pm 0.03$ \\
\hline 2003 SQ317 & 42.62 & 28.60 & 0.07 & $22.73 \pm 0.01$ & $23.29 \pm 0.04$ & $24.56 \pm 0.09$ & $26.12 \pm 0.26$ & $6.84 \pm 0.04$ \\
\hline 2003 UZ117 & 44.42 & 27.42 & 0.13 & $21.42 \pm 0.01$ & $21.94 \pm 0.01$ & $23.41 \pm 0.03$ & $24.63 \pm 0.08$ & $5.47 \pm 0.01$ \\
\hline Sedna (2003 VB12) & 532.7 & 11.92 & 0.85 & $\ldots$ & $\ldots$ & $22.000 \pm 0.009$ & $22.31 \pm 0.01$ & $\ldots$ \\
\hline 2003 WU172 & 39.48 & 4.14 & 0.26 & $21.35 \pm 0.01$ & $21.52 \pm 0.01$ & $22.43 \pm 0.01$ & $22.69 \pm 0.01$ & $6.67 \pm 0.01$ \\
\hline 2004 EH96 & 39.55 & 3.13 & 0.28 & $23.22 \pm 0.02$ & $23.19 \pm 0.02$ & $24.02 \pm 0.04$ & $24.41 \pm 0.05$ & $8.59 \pm 0.02$ \\
\hline 120216 (2004 EW95) & 39.44 & 29.27 & 0.31 & $21.20 \pm 0.01$ & $21.60 \pm 0.01$ & $22.68 \pm 0.01$ & $22.96 \pm 0.01$ & $6.80 \pm 0.01$ \\
\hline 90568 (2004 GV9) & 42.00 & 22.00 & 0.07 & $20.272 \pm 0.008$ & $20.434 \pm 0.007$ & $21.418 \pm 0.007$ & $21.718 \pm 0.009$ & $4.374 \pm 0.008$ \\
\hline 2004 PA112 & 38.88 & 32.97 & 0.10 & $23.23 \pm 0.02$ & $23.58 \pm 0.03$ & $24.38 \pm 0.08$ & $24.69 \pm 0.08$ & $7.79 \pm 0.03$ \\
\hline 2004 QQ26 & 22.91 & 21.45 & 0.14 & $23.24 \pm 0.02$ & $23.60 \pm 0.03$ & $24.55 \pm 0.07$ & $24.83 \pm 0.07$ & $10.16 \pm 0.03$ \\
\hline 2004 TV357 & 48.00 & 9.74 & 0.28 & $22.54 \pm 0.01$ & $23.04 \pm 0.02$ & $24.03 \pm 0.04$ & $24.32 \pm 0.04$ & $7.02 \pm 0.02$ \\
\hline 2004 VN112 & 334.0 & 25.52 & 0.85 & $\ldots$ & $\ldots$ & $24.85 \pm 0.17$ & $24.94 \pm 0.12$ & $\ldots$ \\
\hline 2004 XR190 & 57.74 & 46.56 & 0.10 & $22.18 \pm 0.01$ & $22.46 \pm 0.01$ & $23.46 \pm 0.02$ & $23.73 \pm 0.02$ & $4.55 \pm 0.01$ \\
\hline 2005 CA79 & 48.18 & 11.66 & 0.22 & $21.298 \pm 0.009$ & $21.49 \pm 0.01$ & $22.47 \pm 0.02$ & $22.70 \pm 0.01$ & $5.606 \pm 0.009$ \\
\hline 2005 EZ296 & 39.55 & 1.77 & 0.15 & $22.93 \pm 0.03$ & $22.92 \pm 0.02$ & $23.88 \pm 0.05$ & $24.23 \pm 0.06$ & $7.44 \pm 0.02$ \\
\hline 2005 GB187 & 39.46 & 14.68 & 0.23 & $22.30 \pm 0.01$ & $22.65 \pm 0.02$ & $\ldots$ & $\ldots$ & $7.46 \pm 0.02$ \\
\hline 2005 GE187 & 39.27 & 18.25 & 0.32 & $22.60 \pm 0.02$ & $22.58 \pm 0.01$ & $23.38 \pm 0.02$ & $23.63 \pm 0.02$ & $7.85 \pm 0.01$ \\
\hline 2005 GF187 & 39.39 & 3.90 & 0.25 & $23.09 \pm 0.02$ & $23.49 \pm 0.03$ & $24.67 \pm 0.09$ & $24.79 \pm 0.07$ & $8.30 \pm 0.03$ \\
\hline 2005 PU21 & 175.5 & 6.17 & 0.83 & $\ldots$ & $\ldots$ & $24.13 \pm 0.04$ & $24.43 \pm 0.08$ & $\ldots$ \\
\hline 160427 (2005 RL43) & 24.55 & 12.26 & 0.04 & $22.05 \pm 0.01$ & $22.03 \pm 0.01$ & $22.85 \pm 0.02$ & $23.13 \pm 0.01$ & $8.30 \pm 0.01$ \\
\hline 145452 (2005 RN43) & 41.39 & 19.28 & 0.02 & $20.166 \pm 0.006$ & $20.350 \pm 0.007$ & $21.377 \pm 0.007$ & $21.651 \pm 0.008$ & $4.048 \pm 0.006$ \\
\hline 2005 RO43 & 29.03 & 35.46 & 0.52 & $21.67 \pm 0.01$ & $21.97 \pm 0.01$ & $22.79 \pm 0.01$ & $23.07 \pm 0.01$ & $7.54 \pm 0.01$ \\
\hline 308379 (2005 RS43) & 47.78 & 10.00 & 0.19 & $21.72 \pm 0.01$ & $22.04 \pm 0.01$ & $23.11 \pm 0.02$ & $23.39 \pm 0.02$ & $5.46 \pm 0.01$ \\
\hline $145474(2005$ SA278) & 93.27 & 16.28 & 0.64 & $22.78 \pm 0.02$ & $23.09 \pm 0.02$ & $24.23 \pm 0.07$ & $24.46 \pm 0.05$ & $6.79 \pm 0.02$ \\
\hline $145480(2005$ TB190) & 75.40 & 26.50 & 0.38 & $21.328 \pm 0.009$ & $21.51 \pm 0.01$ & $22.58 \pm 0.01$ & $22.89 \pm 0.01$ & $4.708 \pm 0.009$ \\
\hline 2005 TV189 & 39.63 & 34.35 & 0.19 & $22.97 \pm 0.02$ & $23.25 \pm 0.02$ & $24.07 \pm 0.04$ & $24.32 \pm 0.04$ & $7.98 \pm 0.02$ \\
\hline 2005 VJ119 & 35.28 & 6.95 & 0.68 & $22.15 \pm 0.01$ & $22.53 \pm 0.03$ & $23.27 \pm 0.03$ & $23.63 \pm 0.04$ & $11.48 \pm 0.03$ \\
\hline 2006 HW122 & 45.44 & 1.53 & 0.06 & $23.99 \pm 0.04$ & $24.15 \pm 0.08$ & $24.91 \pm 0.19$ & $25.21 \pm 0.16$ & $7.56 \pm 0.08$ \\
\hline 2006 QH181 & 67.07 & 19.08 & 0.42 & $23.78 \pm 0.04$ & $23.82 \pm 0.04$ & $24.46 \pm 0.06$ & $24.65 \pm 0.08$ & $4.73 \pm 0.04$ \\
\hline 2006 QP180 & 38.01 & 4.95 & 0.65 & $22.72 \pm 0.02$ & $22.62 \pm 0.01$ & $23.30 \pm 0.03$ & $23.65 \pm 0.03$ & $10.15 \pm 0.01$ \\
\hline 308933 (2006 SQ372) & 755.4 & 19.47 & 0.96 & $21.95 \pm 0.01$ & $22.00 \pm 0.01$ & $22.93 \pm 0.02$ & $23.22 \pm 0.02$ & $8.13 \pm 0.01$ \\
\hline 187661 (2007 JG43) & 23.90 & 33.14 & 0.40 & $21.109 \pm 0.009$ & $21.43 \pm 0.01$ & $22.31 \pm 0.01$ & $22.38 \pm 0.02$ & $9.459 \pm 0.009$ \\
\hline 2007 JK43 & 46.14 & 44.91 & 0.48 & $21.254 \pm 0.009$ & $21.46 \pm 0.01$ & $22.32 \pm 0.01$ & $22.61 \pm 0.01$ & $7.472 \pm 0.009$ \\
\hline 225088 (2007 OR10) & 66.84 & 30.92 & 0.50 & $21.66 \pm 0.01$ & $21.41 \pm 0.01$ & $22.05 \pm 0.01$ & $22.47 \pm 0.01$ & $2.33 \pm 0.01$ \\
\hline 2007 RH283 & 15.93 & 21.33 & 0.33 & $21.407 \pm 0.009$ & $21.73 \pm 0.01$ & $22.72 \pm 0.01$ & $23.01 \pm 0.01$ & $8.846 \pm 0.009$ \\
\hline 2007 TA418 & 72.88 & 21.95 & 0.50 & $23.29 \pm 0.02$ & $23.50 \pm 0.03$ & $24.37 \pm 0.08$ & $24.66 \pm 0.08$ & $7.66 \pm 0.03$ \\
\hline
\end{tabular}

Notes.

${ }^{\mathrm{a}}$ MPC designations or names and asteroid numbers (where available). $\mathrm{H}_{606}$ is the absolute magnitude of the object determined from its observed magnitude in the F606w filter and its distance at time of observation.

${ }^{\mathrm{b}}$ F606w and F814w photometry determined from observations presented by Benecchi et al. (2011; see Fraser \& Brown 2012).

large deviations of the source PSF away from those not affected by these issues (see the white points in Figure 1). That image was rejected by our photometry routine. The photometry of the remaining three images differ by less than 0.04 mag, consistent with expectations due to Poisson noise. Repeat visits to each of these non-variable objects demonstrate the absolute consistency of our photometry from visit to visit.

\subsection{Ground Based Observations}

BVRIJHK photometry of 1998 SM165 is reported in Delsanti et al. (2004). In addition, we utilized photometry of 1999 RB216, 1999 TD10, and 2001 QX322 reported in the MBOSS database $^{5}$ (Hainaut \& Delsanti 2002). Colors of 2004 EW95

\footnotetext{
http://eso.org/ ohainaut/MBOSS/MBOSS2.dat
} 


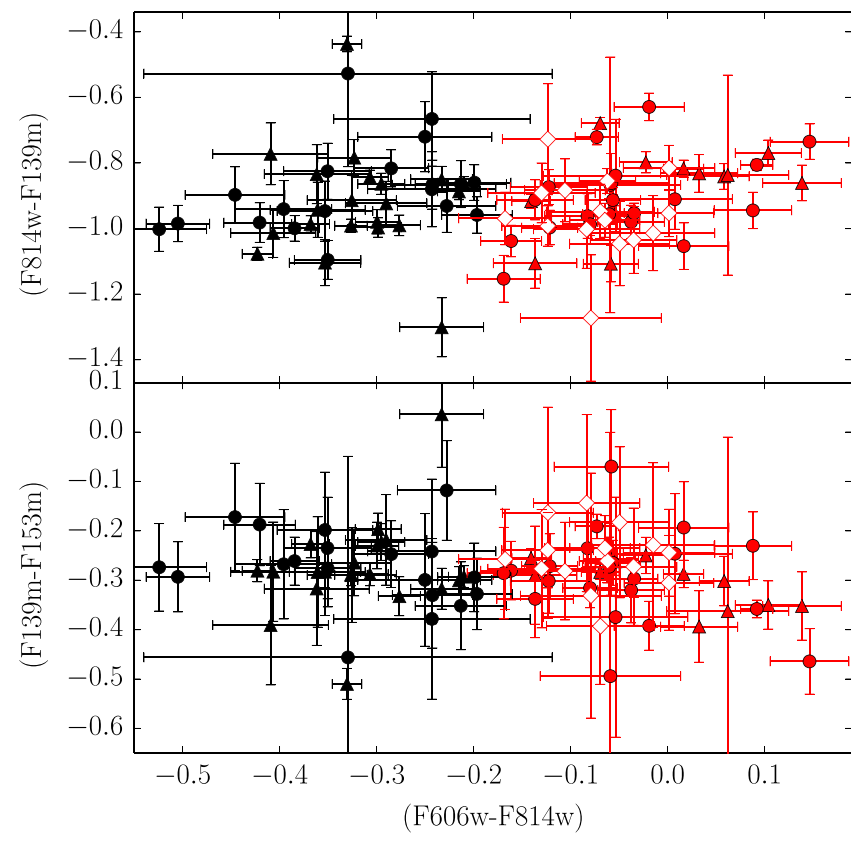

Figure 2. Optical and infrared colors of all cycle 17 targets with $H_{606}>6$. Colors correspond to the neutral (black points) and red (red points) classes identified by the MST clustering. Triangles, circles, and open diamonds represent the excited objects with $q<30 \mathrm{AU}, q>30 \mathrm{AU}$, and the cold classical objects, respectively.

were previously reported in Mommert et al. (2012; $V=21.04 \pm 0.3, \quad(B-V)=0.70 \pm 0.02, \quad(V-R)$ $=0.38 \pm 0.02)$ and Perna et al. $(2013 ; V=21.03 \pm 0.03$, $(B-V)=0.68 \pm 0.04,(V-R)=0.38 \pm 0.04,(R-I)$ $=0.52 \pm 0.05)$.

Unpublished observations of 2004 EW95 were identified with the Solar System Object Image Search tool ${ }^{6}$ provided by the Canadian Astronomy Data Centre (Gwyn et al. 2012) taken at the Very Large Telescope with the FORS2 instrument during 2011 April 2 and 3, in support of the TNOs are Cool program (Perna et al. 2013). The standard calibration images were saturated. Zero-point calibration was possible using ugriz photometry of the background stars observed in the Sloan Digital Sky Survey with conversion to the BVRI systems. ${ }^{7}$ Photometry was extracted using standard aperture photometry techniques, and was ultimately limited by the zero-point calibrations. 2004 EW95 was found to have a visual magnitude of $V=20.86 \pm 0.01$, and colors $(B-V)=0.74 \pm 0.03$, $(V-R)=0.38 \pm 0.04$, and $(R-I)=0.41 \pm 0.07$. Here, we adopt the mean of the observed colors $(B-V)=0.70 \pm 0.03$, $(V-R)=0.38 \pm 0.03$, and $(R-I)=0.46 \pm 0.06$.

\section{RESULTS}

Here, we present the results of our cycle 17 and cycle 18 photometry including a reanalysis of the color classes, and the detection of significant light curve and spectral variations on many of our targets.

\subsection{Compositional Classes}

In Figure 2, we present the colors of the cycle 17 targets with $H_{606}>6$. We apply the same minimum spanning tree (MST)

\footnotetext{
6 http://www3.cadc-ccda.hia-iha.nrc-cnrc.gc.ca/en/ssois/index.html

7 http://sdss.org/dr7/algorithms/sdssUBVRITransform.html
}

and F-statistic-based clustering technique, as well as the same FOP test to the sample as those utilized in Fraser \& Brown (2012). This routine searches for clusters in the data set by pruning a branch of the Euclidean MST, and dividing the sample into two clusters or subsets. The branch that is chosen is that which maximizes the F statistic defined as

$$
F=2\left(\frac{\min \left(\left|t_{1}\right|,\left|t_{2}\right|\right)}{N}\right)\left(\frac{l}{l_{\max }}\right),
$$

where $t_{1}$ and $t_{2}$ are the number of objects in each of the two subpopulations, $N=t_{1}+t_{2}$, and $l$ and $l_{\max }$ are the lengths of the pruned branch and longest branch in the tree, respectively. This method generally selects two subpopulations which are maximally separated in a Euclidian sense. The FOP test then determines the probability of finding a value of $\mathrm{F}$ as large as that observed from an equal-sized sample with similar measurement uncertainty distribution. This is done by creating random samples bootstrapped from the observed colors and uncertainties.

The color distribution bifurcates at $(\mathrm{F} 606 \mathrm{w}-\mathrm{F} 814 \mathrm{w})=0.19$ into the red and neutral classes of object, in agreement with the observed bifurcation of optical colors of the centaurs and dynamically excited objects. The FOP test identifies the bifurcation at greater than $70 \%$ significant while Hartigan's DIP test finds only a $3 \%$ probability that the total color sample of small objects is unimodal.

Our reanalysis repeats the same findings as we previously found, and can be summarized as follows.

1. The dynamically excited KBOs bifurcate into two color classes based on their optical color.

2. Each class exhibits correlated optical and infrared colors. The Spearman rank test gives probabilities of $4 \%$ and $0.6 \%$ that the neutral and red classes do not exhibit correlated colors.

3. The centaurs $(q<30$ AU) and more distant excited objects do not exhibit different color distributions. Whatever apparent differences exist cannot be distinguished from observational biases that affect the sample, including the known trend of visible albedo and color (Stansberry et al. 2008; Fraser \& Brown 2012).

4. The cold classical objects with $41<a<45$ AU and $i<5^{\circ}$ fall on the neutral end of the red class of object. All cold classical objects we observe belong to the red class of object.

The recent work of Dalle Ore et al. (2013) suggests that the next division based on optical and NIR colors would divide the neutral class at $(\mathrm{F} 606 \mathrm{w}-\mathrm{F} 814 \mathrm{w}) \sim-0.35$ and $(\mathrm{F} 814 \mathrm{w}-\mathrm{F} 139 \mathrm{~m})$ $\sim-0.9$. We find no evidence in support of this additional level of complication. On the contrary, as presented below, the colors of 2005 TV189 suggest that the neutral class we identify here is not a sum of overlapping smaller classes, but rather is one continuous class.

\subsection{Brightness Variations}

From comparison of the dual epochs of F606w photometry, it is clear that most of our targets exhibit significant brightness variations. We demonstrate this in Figure 3 where we plot $\left|\Delta m_{606}\right|$, the absolute value of the difference in measured F606w magnitude between cycles 17 and 18. Six of the 12 


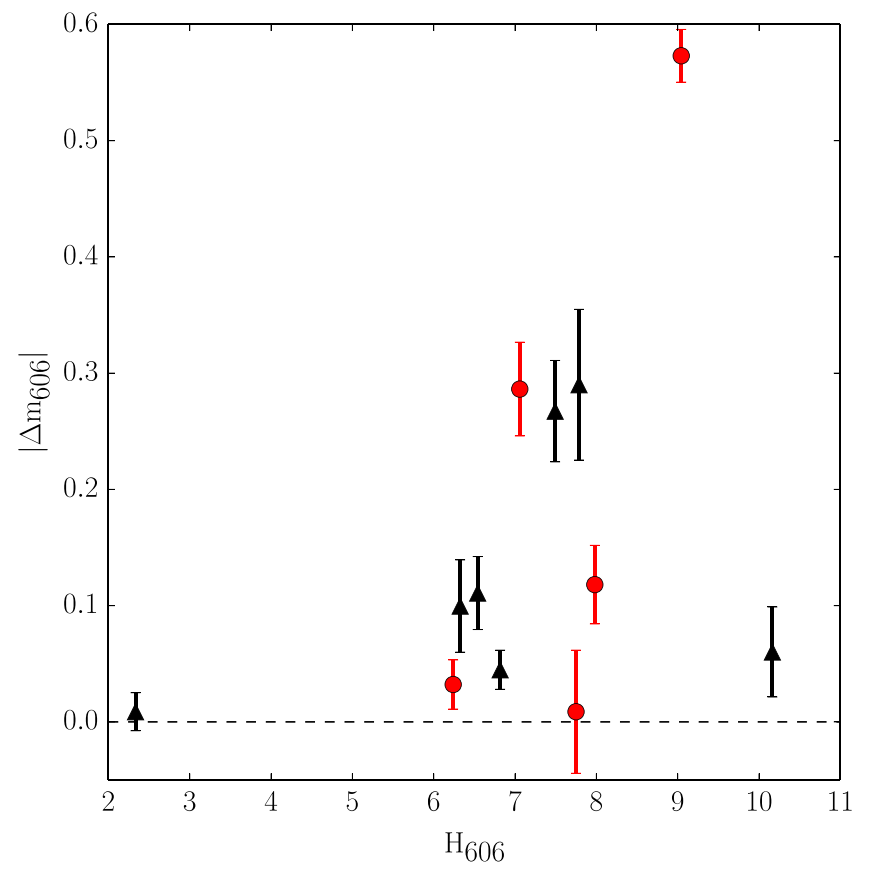

Figure 3. Absolute magnitude of the difference in F606w repeat measurements from cycle 17 and cycle 18 vs. the F606w absolute magnitude of the targets. Red circles and black triangles represent those objects that do and do not exhibit spectral variability, respectively (see Section 3.3).

objects with two F606w measurements exhibit variations in amplitude greater than the $3 \sigma$ uncertainty on the measurement difference. The variations of the measured F606w magnitude between the cycle 17 and cycle 18 visits for those 6 objects range between 0.1 and $0.6 \mathrm{mag}$. We note here that groundbased observations detect a $\sim 0.1$ mag r'-band variation in the brightness of 2004 EW95 that we do not detect here. The consistency of EW95's F606w photometry between the two visits may just be chance, and this object may exhibit significant variability.

It is possible that some of the small magnitude variations are due to the varying phase angle of the targets between observations. Little information about the phase curves of our targets is available. We can, however, estimate the effects of phase using the typical behavior of the few KBOs for which phase information is available; we adopt a linear phase curve with slope $\beta=0.14 \mathrm{mag} \mathrm{deg}^{-1}$ as done in Perna et al. (2013). Comparing the estimated brightness variations to those observed, we see that only the variations of 2005 TV189 can be accounted for. All other observed variations are too large or posses the incorrect sign to be consistent with the phase variations exhibited by other KBOs.

Of the targets with $H_{606}>6,6$ out of 11 present significant brightness variations inconsistent with phase effects. Interestingly, assuming sinusoidal light curves and excluding the large object 2007 OR10 which exhibits no detectable brightness variations, our entire sample is fully consistent with all objects having light curve amplitudes at least as large as 0.26 mag, the mean amplitude of all measured light curves in the Kuiper Belt found by Duffard et al. (2009). That is, our sample is consistent with all small objects having significant light curves. This sample chosen for observations during cycle 18 was selected for their observed cycle 17 colors and not their variability. This suggests that the majority of KBOs smaller than $H_{606} \sim 6$ exhibit light curve variations greater than a couple tenths of a magnitude.

\subsection{Spectral Variations}

Relative reflectance spectra were obtained from the photometry by removing Solar colors from the observed spectra. Solar colors were determined from the synphot routine using Buser-Kurucz stellar analogs. We do not account for an uncertainty of $\sim 0.01$ magnitudes as a result of not having a perfect Solar analog. Our reflectance spectra, normalized to 1 in the F606w filter, are shown in Figures 4-8. For object 2001 QX322, we also present spectra determined from the (F110wF160w) color presented by Benecchi et al. (2011).

It is clear from Figures 6 and 7 that more than half of our targets do not exhibit spectral variations. That is, a smooth, continuous, approximately linear spectrum could simultaneously account for the spectra derived from cycles 17 and 18 , and where available, ground-based photometry. For objects 1998 SM165, 1999 TD10, 2001 PK47, 2001 QX297, and 2005 TV189, this is not the case. We plot the spectra of those objects in Figures 4 and 5.

These five objects appear to exhibit spectral variability. That is, the spectra derived from the cycle 17 photometry is inconsistent with that derived from the cycle 18 photometry. In particular, for SM165, TV189, TD10, and PK47, extrapolation of the cycle $18 \sim 1 \mu \mathrm{m}$ photometry to longer wavelengths appears inconsistent with the cycle 17 F139m measurement. Extrapolation from the cycle $18 \mathrm{~F} 775 \mathrm{w}$ filter to the cycle 17 measurement in the F814w filter reveals a similar inconsistency for 2001 QX297.

We quantify these discrepancies in two ways. A linear fit to the F098m, F110w, and F127m filters from cycle 18 was used to predict the magnitude in the F139m filter. Similarly, a linear fit to the cycle 18 F606w and F775w filters was used to predict the magnitude in the F814w filter. We plot the difference between predicted and measured magnitudes from cycle 17 in Figure 9. As can be seen, 1998 SM165 and 2005 TV189 exhibit a difference between predicted and observed F139m magnitudes that is larger than the $3 \sigma$ uncertainty (considering both observation uncertainty and uncertainty due to the linear fit). 1999 TD10 and 2001 PK47 exhibit greater than $2 \sigma$ variations. Only object 2001 QX297 exhibits a significant difference between predicted and observed F814w magnitudes. Note that we exclude 2007 OR10 from this analysis as it appears to have a spectrum affected by methane absorption, and thus does not exhibit a linear spectrum in the NIR range (Brown et al. 2011).

There are various phenomena that may manifest themselves as the spectral variations we detect. Some of our targets may exhibit large and rapid variations which may affect the photometry in different filters, and thus appear as spectral variations. We searched for such effects in our observations by removing the average observed colors from the non-F606w photometry to provide estimated F606w photometry throughout the visit to a source. This was then plotted against time to search for temporal trends. Some hints of brightness variations throughout an orbit were detected, but not of an amplitude sufficient to account for the $20-50 \%$ spectral variations we report here. 


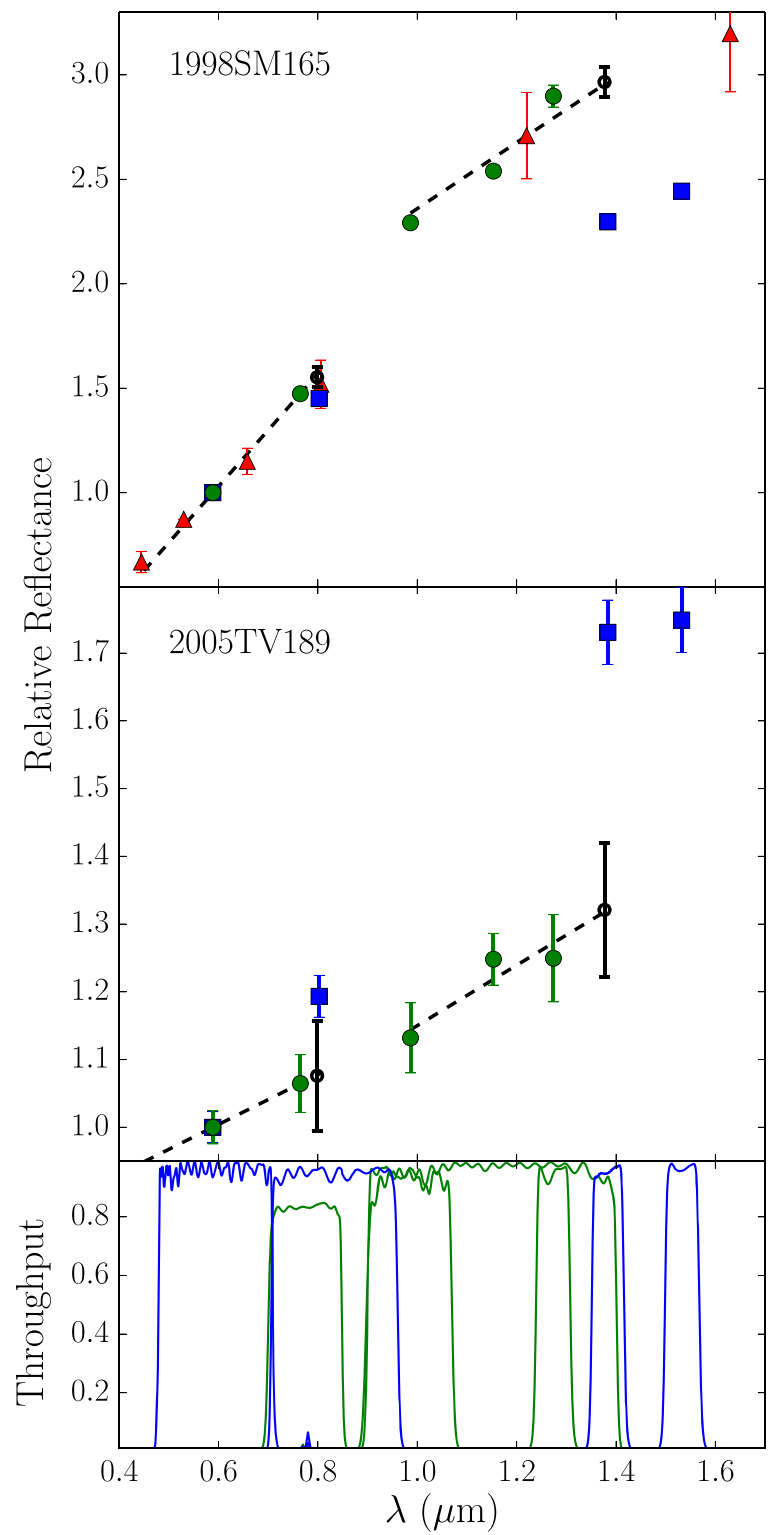

Figure 4. Reflectance spectra of the spectrally variable objects 1998 SM165 and 2005 TV189. Spectra have been normalized to the F606w filter. Blue squares and green circles mark the spectra derived from the cycle 17 and cycle 18 photometry, respectively. Black open circles represent the estimated F814w and $\mathrm{F} 139 \mathrm{~m}$ photometry from the linear regression of the cycle 18 optical (F606w and F775w) and NIR (F098m, F110w, F127m) photometry, which are shown by the dotted lines. The filter bandpasses used in the cycle 17 and 18 photometry are shown by the blue and green curves, respectively, in the bottom panel. Red triangles are spectra derived from ground-based observations.

It may be the case that some previously undetected very sharp and very deep absorption features are causing the discrepancies we observe. While we cannot formally eliminate the existence of deep and sharp features as the source of the apparent spectral variability on these objects, that possibility seems unlikely, as the features required would be so sharp and deep as to be incompatible with typical solid state absorption features. The apparent spectral variability also is not due to sensitivity issues. As discussed above, the WFC 3 filter zero points varied by less than $1 \%$ across the cycles during which the data we utilize were taken (Kalirai et al. 2011). As such, calibrations cannot be the cause of the apparent spectral

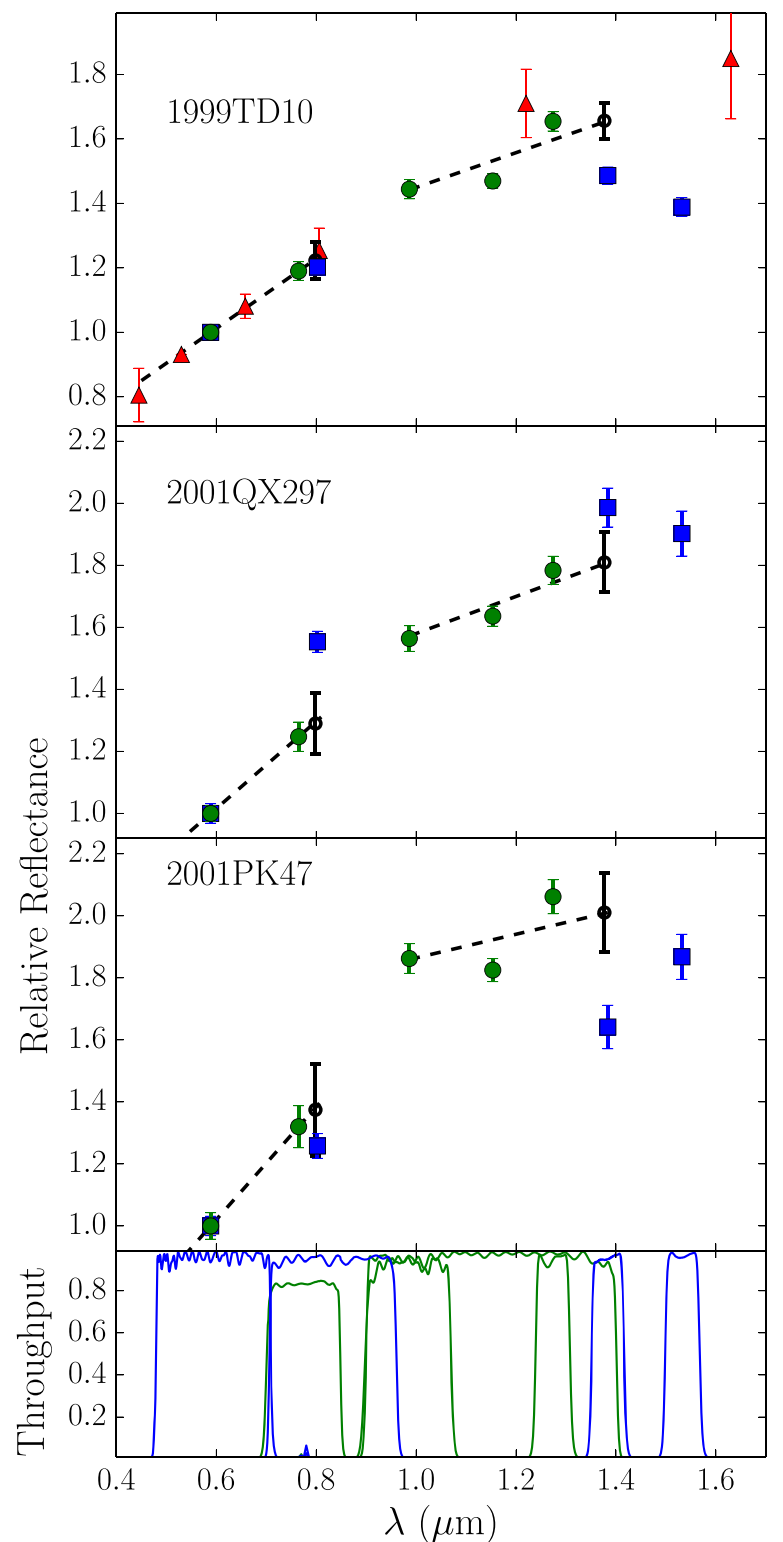

Figure 5. As in Figure 4, but for objects which show $2 \sigma$ spectral variations.

variation. It appears that these five objects truly exhibit spectral variability.

We should note that our detected spectral variability of 1999 TD10 is consistent in theme with the findings of Choi et al. (2003), who found that TD10 exhibits significant optical color variations with amplitude large enough to be apparent in our data. Our observations do not corroborate their results, however, as we find no significant optical variations. Like Choi et al. (2003), we find that 1999 TD10 exhibits significant light curve variation of $\sim 0.6 \mathrm{mag}$. Drawing from their measured light curves, our observations happen to fall near a peak and trough of TD10's variability, and as such should exhibit a $\sim 0.3 \mathrm{mag}$ variation in (F606w-F814w) between the two visits. This variation would be easily detected with the precision of the measurements we present here, and as such our results are at odds with those of Choi et al. (2003). It may be that their variations are entirely due to undetected cometary activity, which could explain the bluer-than-Solar colors they observed. The cause of this discrepancy, however, remains unknown. 


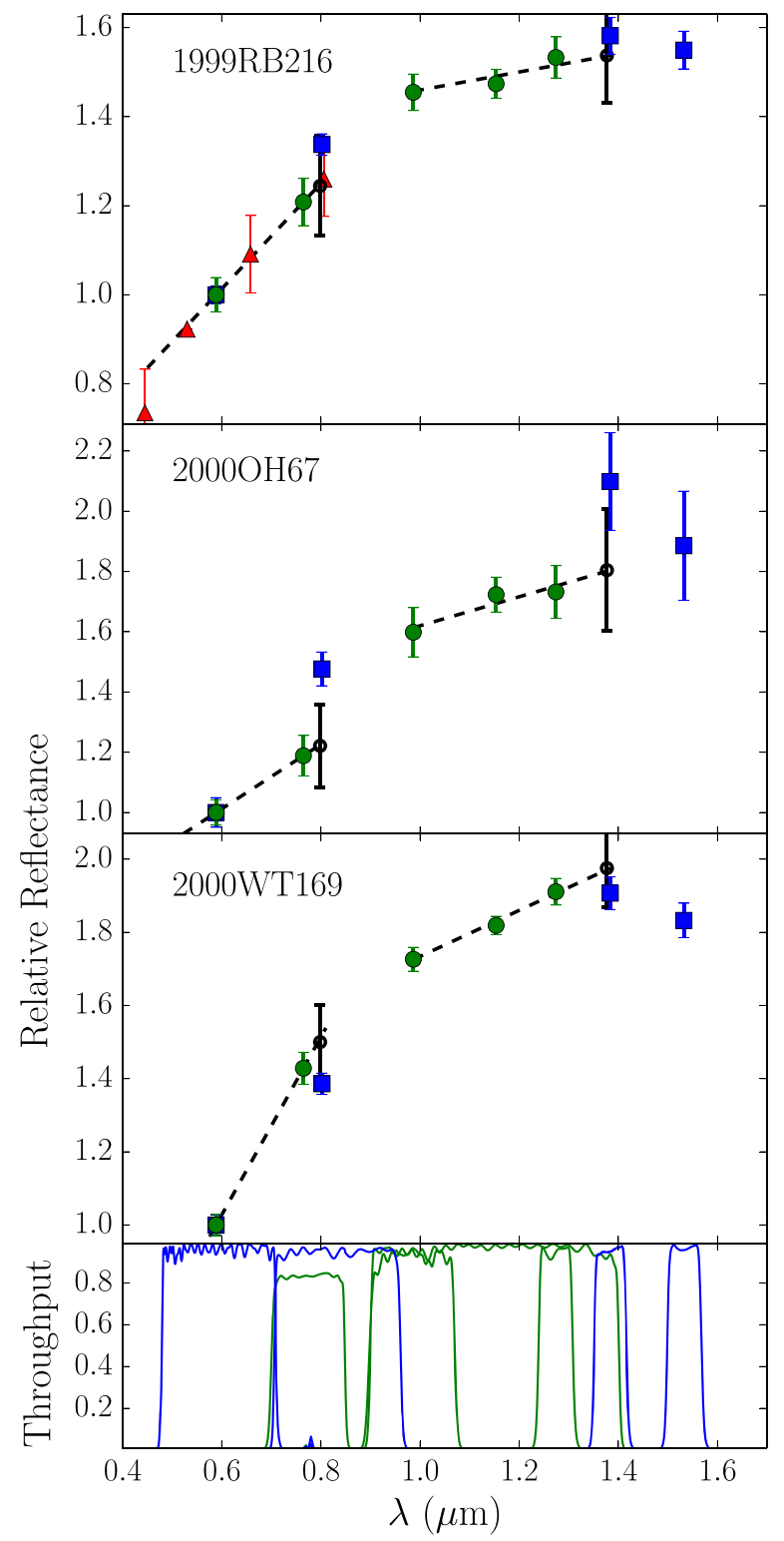

Figure 6. As in Figure 4, but for objects which show no spectral variations.

\section{DISCUSSION}

The objects in our sample that exhibit statistically significant light curves exhibit a broad range of variability with $0.1 \lesssim\left|\Delta m_{606}\right| \lesssim 0.6$. Our sample objects with $H_{606}>6$ are all consistent with having variability with amplitude greater than $0.26 \mathrm{mag}$, that is, the mean of the light curve amplitudes found by Duffard et al. (2009). Not all of the objects with detected brightness variations have associated spectral variations. In the Kuiper Belt, the reddest objects have higher albedos than neutral colored objects (Stansberry et al. 2008; Fraser \& Brown 2012; Lacerda et al. 2014). Assuming the materials responsible for the observed spectral variations are not unique to those objects but are the same material found on other KBOs, then it follows that the brightness variations of all TNOs are primarily shape driven, and not a result of compositional variations. Our observations suggest that the majority of objects with $H_{606}>6$ posses highly non-spherical

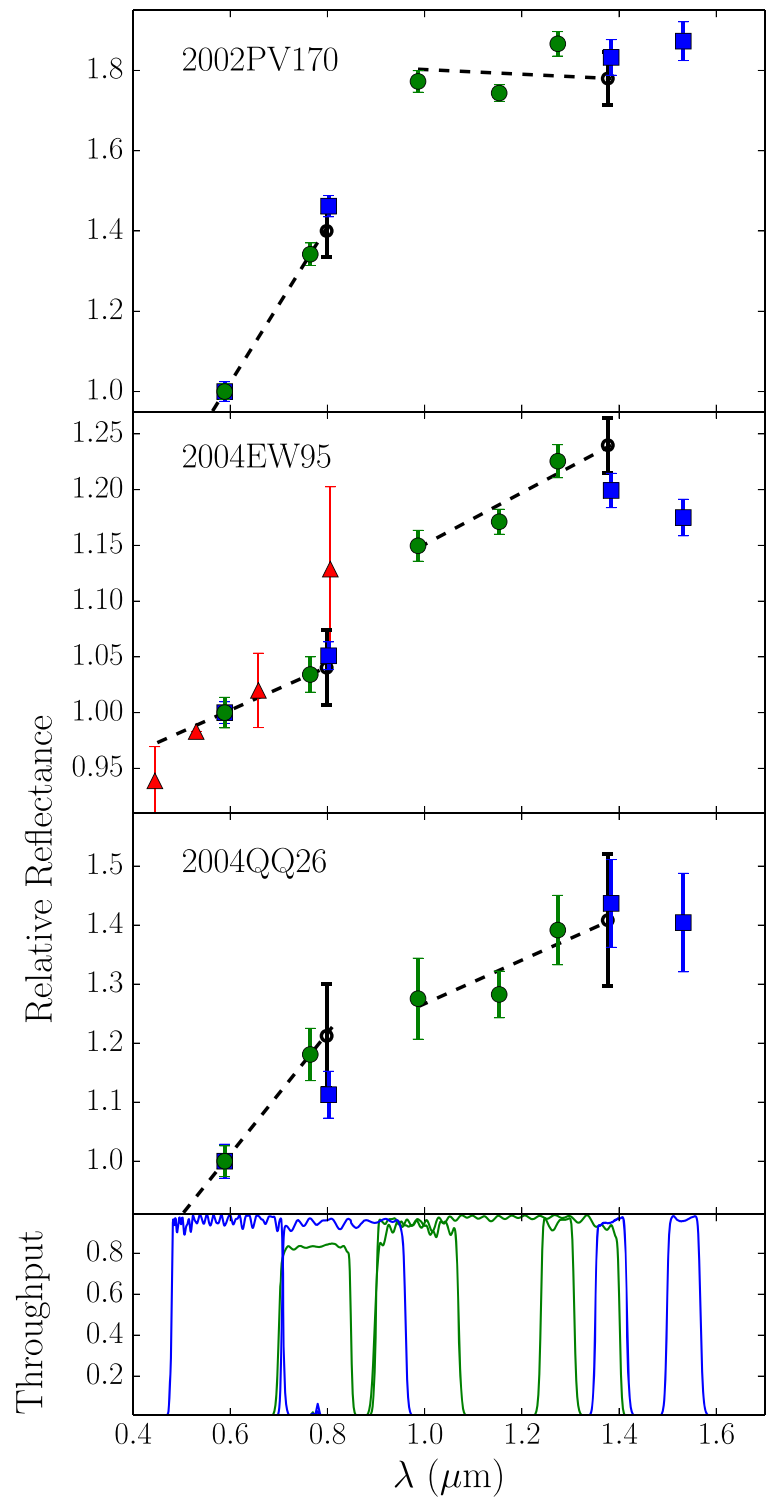

Figure 7. As in Figure 6.

shapes. We caution, however, that the targets were chosen for their color properties and do not exhibit an unbiased sample. Therefore, our statements on the variability as a function of $H$ should be taken with appropriate caution.

Of our sample, five exhibit spectral variations. These five objects occupy a broad range of properties. They span essentially the entire range of dynamical classes: 1998 SM165 belongs to the scattered disk, 2005 TV189 is in a mean-motion resonance with Neptune, 2001 PK47 is a hot classical object, 1999 TD10 is a centaur (a scattered disk object in the system of Gladman et al. 2008), and 2001 QX297 is a cold classical as we defined them in Fraser \& Brown (2012). They span a factor of $\sim 4$ in size, having absolute magnitudes $6 \leqslant H_{606} \leqslant 9$. They occupy the full range of KBO colors from near Solar colored to extremely red. Finally, they exhibit a broad range of light curve amplitudes. 2001 PK47 does not exhibit a significant light curve, while 1999 TD10 exhibits a large $\sim 0.3 \mathrm{mag}$ amplitude. Interestingly, those objects that do not exhibit spectral variability occupy the same range of properties. This suggests that whatever the source of the 


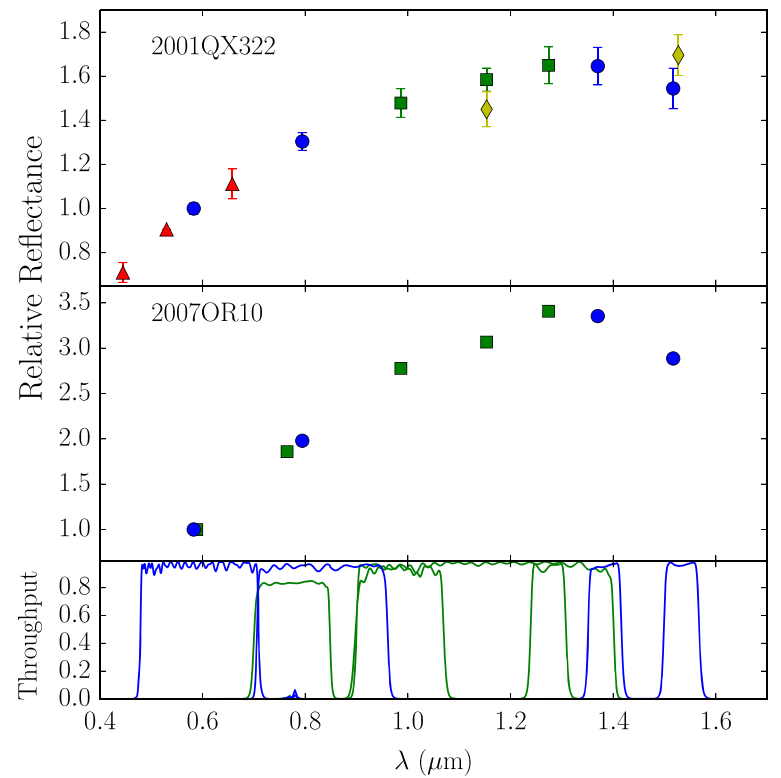

Figure 8. As in Figure 6. The yellow diamonds mark the spectra obtained for 2001 QX322 from the data presented by Benecchi et al. (2011). The F098m, F110w, and F127m photometry of 2001 QX322 have been vertically scaled for visible representation to match the F139m photometry and the photometry of Benecchi.

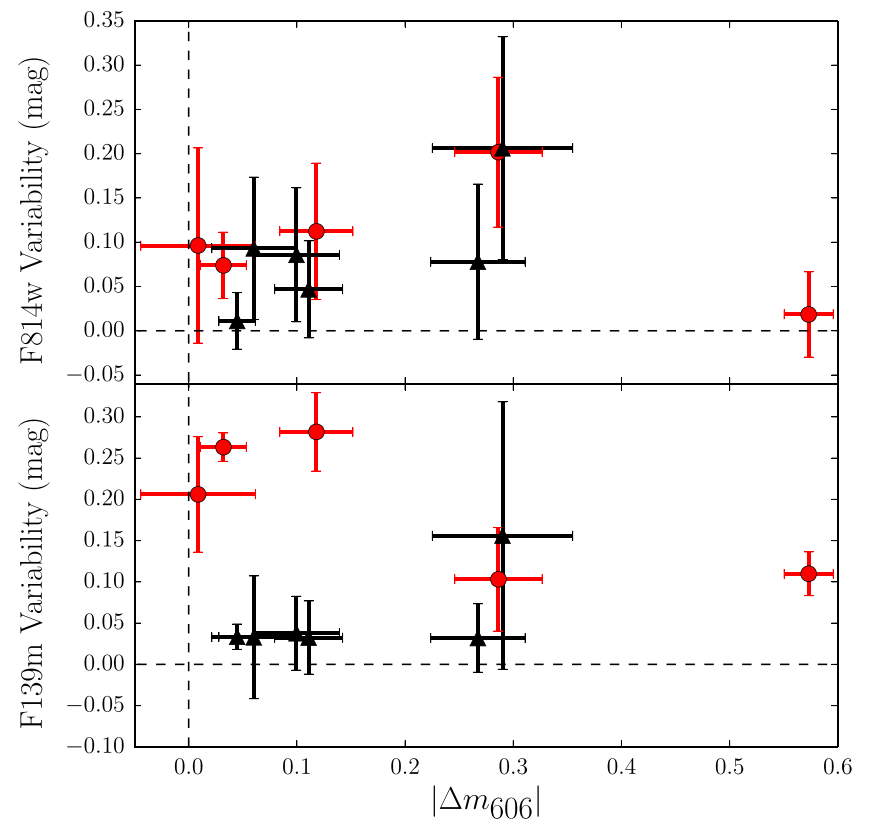

Figure 9. Spectral variability vs. absolute magnitude of the difference in F606w repeat measurements from cycle 17 and cycle 18. Top: variability in the F814w filter. Bottom: variability in the F139m filter. Red circles and black triangles represent those objects that do and do not exhibit spectral variability respectively.

spectral variation, it cannot be driven purely by shape, size, or thermal environment.

One clue to the nature of the spectral variability and the color distribution comes from the (F606w-F814w) and (F814wF139m) variability. We present this in Figure 10. From its cycle 17 and model photometry, 2001 PK47 has an ambiguous classification, having an optical color compatible with either red or neutral classes. 2001 QX297, a cold classical, and hence belonging to the red class, also has ambiguous model color

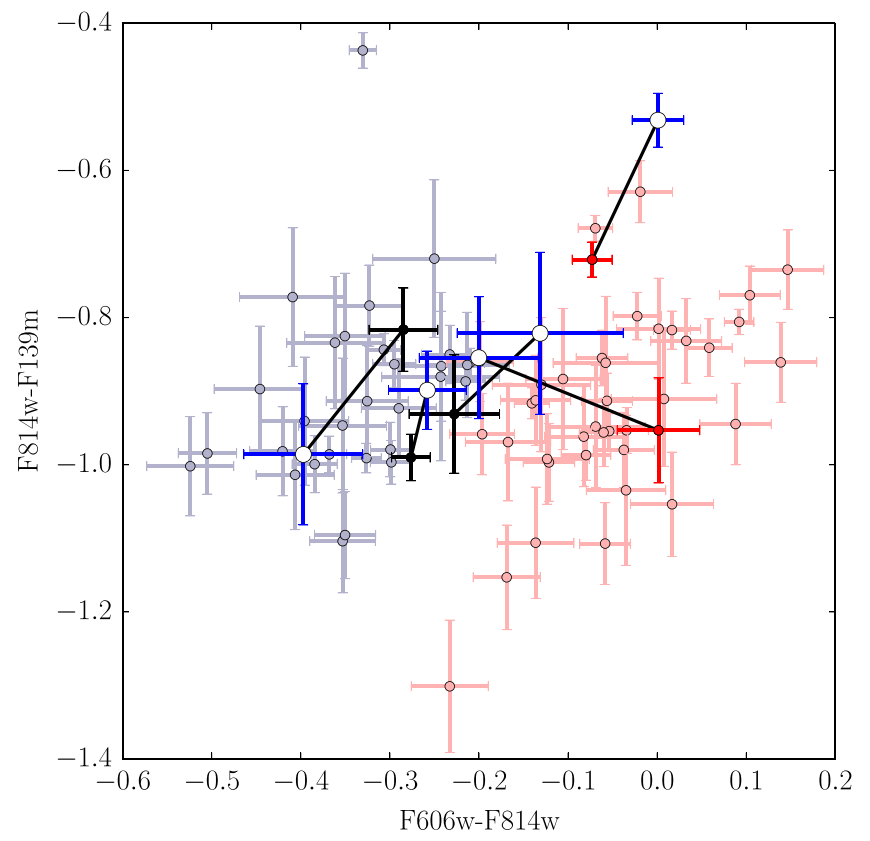

Figure 10. Observed and model optical and infrared colors of the five spectrally variable objects. The model cycle 18 colors are shown in blue points, while the observed cycle 17 colors are shown as solid red or black points according to their cycle 17 classification. Black lines connect the observed and model colors for each source. Gray and light red points show the full cycle 17 colors for comparison.

classification with model colors sufficiently uncertain to $($ F606w-F814w $)=-0.19$ the color division between the red and neutral classes. Objects 1998 SM165, 1999 TD10, and 2005 TV189 all have sufficiently accurate model colors for classification. What is immediately apparent from Figure 10 is that the classifications for those three objects are the same between the two visits. That is, no object observed as red in cycle 17 was found to be neutral in cycle 18 , and vice versa.

Equally as interesting as the consistency of classification, the difference in model colors for TV189 and TD10 causes each to span a broad range in both optical and infrared colors. TV189 in particular spans nearly the full range occupied by the neutral class. This argues strongly in favor of the neutral class being one continuous class rather than the overlap of underlying, more compact classes. The reasoning is simple; it must be that at times, TV189 exhibits colors intermediate between the two extremes we have observed. Thus, the neutral class, of which TV189 is a member, spans at least the range between the two observed colors.

Another way to envision this is to consider the scenario in which the neutral class $((\mathrm{F} 606 \mathrm{w}-\mathrm{F} 814 \mathrm{w})<-0.19)$ is made up of two populations, each possessing different optical and NIR colors. Furthermore, consider the possibility that TV189 is the product of a merger of one object of each of those populations. It then must be that these mergers occur frequently enough such that one of the only two spectrally variable neutral objects we observe is the product of such a merger. In this scenario, the two populations would be mixed together by these mergers, and would no longer be well defined in a color-color plot. Rather, they would appear as one broad class with some redder objects, some more neutral objects, and some objects having intermediate colors between the two. This is exactly the scenario we put forth in Fraser \& Brown (2012) to describe the 
compositional classes-a class' members are mixtures of different materials. We have insufficient data to draw similar conclusions for the red sample. It appears, however, that the neutral class is one class spanning a broad range of colors, rather than multiple discrete classes occupying smaller color ranges.

As discussed above, it seems the classes cannot be due to thermal environment. Furthermore, processes such as cometary activity or collisions, if dominant, would likely homogenize the colors of a class' members. As such, large variations like that of TV189 would not persist. Collisions can be further excluded as the source of the variability when one considers the collisional environment. Dell'Oro et al. (2013) demonstrated that collisions in the Kuiper Belt typically occur at $1-2 \sim \mathrm{km} \mathrm{s}^{-1}$, meaning that most collisions are destructive rather than constructive, which is a requirement to deposit a large amount of differently colored material on an object.

The most obvious conclusion from this result is that at least the members of the neutral class possess a broad range of compositions. This is a natural outcome of the mixing model put fourth by Fraser \& Brown (2012), in which the colors of a class are mainly determined by a mixture of materials unique to that class, under the assertion that some objects, like 2005 TV189, do not posses uniform surface compositions. Further confirmation of both the model and the classifications proposed by Fraser \& Brown (2012) will come from additional, more accurate spectral variation measurements. In particular, it will be extremely interesting to test if the red class is one continuous class, as we have shown for the neutral class with TV189 and TD10, or further bifurcates into smaller subclasses. In addition, further measurements should be made to determine if objects do vary back and forth across the optical color division between the red and neutral classes. If confirmed, this unexpected discovery would largely challenge the existence of two separate classes and would require a broad rethinking of our understanding of the compositions of small KBOs.

\section{REFERENCES}

Barucci, M. A., Belskaya, I. N., Fulchignoni, M., \& Birlan, M. 2005, AJ, 130, 1291

Barucci, M. A., Boehnhardt, H., Dotto, E., et al. 2002, A\&A, 392, 335

Benecchi, S. D., Noll, K. S., Stephens, D. C., Grundy, W. M., \& Rawlins, J. 2011, Icar, 213, 693

Brown, M. E., Burgasser, A. J., \& Fraser, W. C. 2011, ApJL, 738, L26

Bus, S. J., Vilas, F., \& Barucci, M. A. 2002, in Asteroids III, ed. W. F. Bottke Jr. et al. (Tucson, AZ: Univ. Arizona Press), 169

Choi, Y. J., Brosch, N., \& Prialnik, D. 2003, Icar, 165, 101

Dalle Ore, C. M., Dalle Ore, L. V., Roush, T. L., et al. 2013, Icar, 222, 307 de Bergh, C., Boehnhardt, H., Barucci, M. A., et al. 2004, A\&A, 416, 791

Dell'Oro, A., Campo Bagatin, A., Benavidez, P. G., \& Alemañ, R. A. 2013, A\&A, 558, A95

Delsanti, A., Hainaut, O., Jourdeuil, E., et al. 2004, A\&A, 417, 1145

Duffard, R., Ortiz, J. L., Thirouin, A., Santos-Sanz, P., \& Morales, N. 2009, A\&A, 505, 1283

Fornasier, S., Barucci, M. A., de Bergh, C., et al. 2009, A\&A, 508, 457

Fornasier, S., Doressoundiram, A., Tozzi, G. P., et al. 2004, A\&A, 421, 353

Fraser, W. C., \& Brown, M. E. 2012, ApJ, 749, 33

Gladman, B., Marsden, B. G., \& Vanlaerhoven, C. 2008, in The Solar System Beyond Nepture, ed. M. A. Barucci et al. (Tucson, AZ: Univ. Arizona Press), 43

Gwyn, S. D. J., Hill, N., \& Kavelaars, J. J. 2012, PASP, 124, 579

Hainaut, O. R., \& Delsanti, A. C. 2002, A\&A, 389, 641

Kalirai, J. S., Deustua, S., Rajan, A., \& Riess, A. 2011, The Photometric Performance of WFC3/IR: Temporal Stability Through Year 1, Tech. Rep.

Krist, J. 1993, in ASP Conf. Ser. 52, Astronomical Data Analysis Software and Systems II, ed. R. J. Hanisch, R. J. V. Brissenden, \& J. Barnes (San Francisco, CA: ASP), 536

Lacerda, P., Fornasier, S., Lellouch, E., et al. 2014, ApJL, 793, L2

Lacerda, P., Jewitt, D., \& Peixinho, N. 2008, AJ, 135, 1749

Licandro, J., \& Pinilla-Alonso, N. 2005, ApJL, 630, L93

Merlin, F., Barucci, M. A., de Bergh, C., et al. 2010, Icar, 208, 945

Merlin, F., Barucci, M. A., Dotto, E., de Bergh, C., \& Lo Curto, G. 2005, A\&A, 444, 977

Mommert, M., Harris, A. W., Kiss, C., et al. 2012, A\&A, 541, A93

Peixinho, N., Delsanti, A., Guilbert-Lepoutre, A., Gafeira, R., \& Lacerda, P. 2012, A\&A, 546, 86

Perna, D., Dotto, E., Barucci, M. A., et al. 2013, A\&A, 554, A49

Stansberry, J., Grundy, W., Brown, M., et al. 2008, in The Solar System Beyond Nepture, ed. M. A. Barucci et al. (Tucson, AZ: Univ. Arizona Press), 161

Tegler, S. C., \& Romanishin, W. 2003, Icar, 161, 181

Tegler, S. C., Romanishin, W., \& Consolmagno, G. J. 2003, ApJL, 599, L49 